\title{
Clathrate Superhydrides Under High Pressure Conditions: A Class of Extraordinarily Hot Conventional Superconductors
}

\author{
Yanming Ma \\ College of Physics, Jilin Univ., Changchun 130012, China; \\ mym@jlu.edu.cn
}

Room-temperature superconductivity has been a century long-held dream of mankind and a focus of intensive research. Recent progress on findings of room-temperature superconductors among superhydrides stabilized at high pressure conditions is remarkable. Focus is placed on a class of clathrate superhydrides, the best ever-known family of superconductors, that exhibit extraordinarily high$T_{\mathrm{c}}$ superconductivity (e.g., $T_{\mathrm{c}}=260 \mathrm{~K}$ for $\mathrm{LaH}_{10}[1-4]$ ).

The first-ever clathrate structure in superhydride is proposed in $\mathrm{CaH}_{6}[5]$ by my group that shows a potential of high$T_{\mathrm{c}}$ superconductivity at about $235 \mathrm{~K}$. This clathrate structure accepts the emergence of unusual $\mathrm{H}$ cages, in which $\mathrm{H}$ atoms are weakly covalently bonded to one another, with $\mathrm{Ca}$ atoms occupying the centers of the cages. The high- $T_{\mathrm{c}}$ superconductivity is arising from the peculiar H clathrate structure.

We recently found a common rule of the formation of superconducting clathrate structures in rare earth (RE, e.g., Sc, Y, La, Ce, Pr., etc) superhydrides accompanying the occurrence of three different stoichiometries of $\mathrm{REH}_{6}, \mathrm{REH}_{9}$, and $\mathrm{REH}_{10}$, some of which exhibit extraordinarily high- $T_{\mathrm{c}}$ superconductivity [1]. Subsequent experiments [3,4,6,7] indeed synthesized the as-predicted clathrate superhydrides $\mathrm{YH}_{6}, \mathrm{YH}_{9}$, and $\mathrm{LaH}_{10}$ with measured $T_{\mathrm{c}}$ values at 224,243 , and $260 \mathrm{~K}$, respectively, setting up new $T_{\mathrm{c}}$ records among known superconductors. These discoveries open the door of achieving superconductors that could work at room temperature (300 K) in superhydrides.

In the talk, I will give an overview on the status of research progress on superconductive superhydrides, and then discuss the design principle for achieving room-temperature superconductor. Our prediction on a hot superconductor $\left(T_{\mathrm{c}}\right.$ at $\left.\sim 400 \mathrm{~K}\right)$ in a clathrate superhydride $\mathrm{Li}_{2} \mathrm{MgH}_{16}$ [8] together with future research direction will be discussed.

[1] Peng et al., PRL 119, 107001 (2017).

[2] Liu et al., PNAS 114, 6990 (2017).

[3] Somayazulu et al., PRL 122, 027001 (2019).

[4] Drozdov et al., Nature 569, 528 (2019).

[5] Wang et al., PNAS 109, 6463 (2012).

[6] Kong et al., arXiv 1909.10482 (2019) ; Snider et al., PRL 126, 117003 (2021).

[7] Troyan et al., Adv. Mater. 33, 2006832 (2021).

[8] Sun et al., PRL 123, 097001 (2019).

Keywords: High-Tc superconductivity, Clathrate structure, Superhydride 